

\title{
Public versus Private Insurance with Non-Expected Utility: a Political Economy Argument
}

\author{
Jean Hindriks*
}

July 2001

\begin{abstract}
This paper analyzes the political support for public insurance in the presence of a private insurance alternative. The public insurance is compulsory and offers a uniform insurance policy. The private insurance is voluntary and can offer different insurance policies. Adopting Yaari's (1987) dual theory to expected utility (i.e., risk aversion without diminishing marginal utility of income), we show that adverse selection on the private insurance market may lead a majority of individuals to prefer public insurance over private insurance, even if the median risk is below the average risk (so that the median actually subsidizes high-risk individuals). We also show that risk aversion makes public insurance more attractive and that the dual theory is less favourable to a mixed insurance system than the expected utility framework. Lastly, we demonstrate how the use of genetic tests may threaten the political viability of public insurance.
\end{abstract}

Keywords:Voting, insurance, adverse selection.

JEL classification: H51, H23

* Economics department, Queen Mary,University of London, Mile End Road, London E1 4NS, England. Tel. (44)-020-78827807, Email:j.hindriks@qmw.ac.uk. 


\section{Introduction}

This paper is concerned with the political demand for public insurance when there exist private insurance alternatives. We focus attention on the insurance against the risk of incurring a damage, and to keep the argument clear, we abstract from the possible redistributive element in public insurance. ${ }^{1}$ To separate redistribution from insurance arguments, we assume that individuals differ only with respect to their risk (or probability of incurring a damage) without distinguishing the nature of the risk. Therefore we can interpret the analysis among other as regarding the provision of health care insurance when individuals differ in their health status, or the provision of pension annuities when individuals differ in their life expectancy.

There is strong evidence that in the OECD countries the public sector plays an important role in the provision of insurance for health care (see OECD, $1994)^{2}$, and that the retired depend largely on state pensions (Miles and Timmermann, 1999). In most European countries, state pensions are typically 50-80 percent of average earnings. The essential distinction we make between private insurance and public insurance is that private insurance is voluntary and can offer different insurance policies (essentially, premia and coverage rates) to different individuals while public insurance is compulsory and offer the same insurance contract to everyone. There are two good reasons why public insurance is compulsory. First, to overcome the adverse selection problem which induces the good risks to opt out increasing the average cost

\footnotetext{
${ }^{1}$ We can motivate this assumption on the basis of recent empirical work by Lynn Coronado and al (2000) showing that social security has negligible distributive effects.

${ }^{2}$ More recently, the Economist (July 16th 2001) reports a study by the OECD according to which in 1998 Germany spent $10 \%$ of GDP on health, France spent 9.5\%, the Netherlands spent 8.7\%, Italy spent 8.2\%, Austria spent $8 \%$ and Britain spent just $6.8 \%$. Most-but not all- of health spendings were accounted for by the state: Germany spent $8 \%$, France $8.5 \%$, the Netherlands $6 \%$, Italy $5.5 \%$, Austria $5.8 \%$ and Britain $5.7 \%$.
} 
of insurance. Second, to avoid the so-called Samaritan's dilemma problem according to which if individuals anticipate that the government will provide them with insurance whether or not they insure, this will undermine their incentive to buy insurance.

Focusing on the pure insurance element, we derive the majority rule equilibrium between private and public insurance. We use the Rothschild and Stiglitz (1976) model that we adapt to a continuum of risk types and nonexpected utility framework (i.e., risk aversion without diminishing marginal utility of income). We proceed in two steps. First we derive the political equilibrium outcome when individuals must choose between a public insurance or a private insurance as exclusive to each other (both involving complete coverage). We obtain the result that for a large family of distributions of risks in the population, a majority will prefer public insurance over private insurance, and that the size of this majority is increasing with risk aversion. In that sense no distributive argument is required to justify the provision of public insurance. In a second step, we consider the possibility for individuals to supplement a (basic) public insurance with a private insurance and individuals can vote over the degree of public insurance. The main result we obtain is that for any distribution of risk in the population, mixing public with private insurance is politically non sustainable in the sense that there is always a majority that would like to push this supplementary system towards either a pure public insurance or a full private insurance. This political impossibility of mixing public with private insurance may seem to contradict the well-known argument that it is always desirable to have some public insurance because it operates a cross-subsidy from low-risk to highrisk individuals which is beneficial to both types in relaxing the incentive compatibility constraint on the private insurance market (see Mas-colell and al., 1995, p.459; or Eckstein and al., 1985). While this result is correct in the expected utility setting, our result simply shows that it does not extend to 
our non-expected utility model.

This paper belongs to recent theoretical work on the politics of publicly provided private goods (see, among others, Gouveia, 1995, and Epple and Romano, 1996). However the models used are different as well as the results. For instance this literature adopts the expected utility approach and typically obtains a majority equilibrium involving a mixture of private and public insurance. We may also get a majority support for a mixed system within our non-expected utility framework but by assuming that public insurance is distortionary and that the deadweight loss increases sufficiently rapidly with the level of public insurance (see Appendix B).

The paper is organized as follows. Section 2 deals with voting over exclusive private and public insurance when the private market can only offer a pooling contract. Section 3 introduces the possibility for the private market to offer different contracts to different individuals. Section 4 presents the case where individuals can supplement public insurance with a separating insurance contract and individuals must vote over the degree of public insurance. Section 5 investigates how the demand for public insurance changes when the private insurance companies can use some tests that help them to infer, at least partially, individual risks. Section 6 concludes the paper.

\section{Private insurance without screening}

We use a simple model from Hindriks and Myles (Chapter 12, forthcoming). We consider that there is a large number of risk neutral insurance companies and that the insurance market is competitive. The insurance premium is based on the level of expected risk (no screening) among those who accept insurance offers (voluntary insurance). Competition ensures that profits are 
zero in equilibrium through entry and exit. That is, if there is any new insurance contract that can make a positive profit given the contracts already available, then one of the companies will choose to offer it.

The demand for insurance comes from a large set of risk averse individuals. These can be broken down into a continuum of different types of individuals who differ in their probability of incurring (fixed) damage of (uniform) value $d=1 .^{3}$ The probability of damage for an individual is given by $\theta$. Different types of individuals have different values of $\theta$ lying between 0 and $\Theta$ (with $\Theta<1$ ). There is no moral hazard since individuals cannot affect their probability of accident which is fixed and since the damage is also fixed there is no agency cost related to the fact that for example in health care provision the prescribing agents may have an incentive to prescribe treatment beyond what is necessary for the insured and uninformed patients. ${ }^{4}$ However adverse selection is introduced by assuming that each individual knows their own risk (i.e. value of $\theta$ ) but that it is not observable by the insurance companies. The insurance companies know correctly that risks are distributed in the population on the interval $[0, \Theta]$ according to the cumulative distribution function $F(\theta)$. So $F(\theta)$ is the fraction of the population with probability of accident less or equal to $\theta$. The mean risk is $\bar{\theta}=\int_{0}^{\Theta} \theta d F(\theta)$ and the median risk is $\theta_{m}=F^{-1}(1 / 2)$. We allow both for positively skewed $\left(\theta_{m}<\bar{\theta}\right)$, symmetric $\left(\theta_{m}=\bar{\theta}\right)$ and negatively skewed $\left(\theta_{m}>\bar{\theta}\right)$ distributions.

Since all of the individuals are risk averse, they are willing to pay an insurance premium in excess of their expected damage to avoid facing the cost of damage. The reservation premium that each type $\theta$ is willing to pay for a

\footnotetext{
${ }^{3}$ The current cost escalation in health care can be represented by an increase in $d$ which is easily seen to have no effect on the relative desirability of public and private insurance

${ }^{4}$ Moral hazard and agency cost are assumed away because they affect private and public insurance in the same way and so they should not influence the choice between the two systems.
} 
coverage rate $\delta \in[0,1]$ of a damage $d=1$ is given by

$$
\pi(\theta)=(1+\alpha) \theta \delta
$$

where $\alpha \geq 0$ measures the degree of risk aversion. In Appendix A we show how this reservation premium can be rationalized under Yaari's Dual Theory of choice under uncertainty. The dual theory has the property that utility is linear in income, and risk aversion is expressed entirely by a transformation of probabilities in which bad outcomes are given relatively higher weights and good outcomes are given relatively lower weights. In our simple two-state model the probability $\theta$ of bad outcome is weighted up by the loading factor $(1+\alpha)$ to give $(1+\alpha) \theta>\theta$. To guarantee that this weighted probability is less than 1 for all types $\theta \in[0, \Theta]$, it is assumed that $\alpha \leq \frac{1-\Theta}{\Theta}$. As we shall see this formulation of risk aversion without diminishing marginal utility allows the derivation of a rich set of insights. Although most of the classical results in insurance theory appear to be robust to such departures from the expected utility model, one important implication to the demand of insurance deserves to be emphasized and will play a major role in the rest of the analysis: under the dual theory a risk averse individual will either buy full insurance or no insurance (corner solutions) whereas under expected utility a risk averse individual will always buy partial insurance, unless insurance is actuarially fair. ${ }^{5}$ It would be absurd to suggest that the dual theory provides a better model than the expected utility. The latter has obvious appeal and has provided so many useful results in insurance theory. Nonetheless, we feel there is some gain from studying the properties of our simple nonexpected utility model, even if only to derive some clear insights on the political demand for public insurance and the desirability of a mixed system.

\footnotetext{
${ }^{5}$ An analysis of the effect of non-expected utility models on standard optimal insurance results appears in Doherty and Eeckhoudt (1995) and Machina (1995).
} 
The assumption of competitive insurance market implies that in equilibrium insurance companies must earn zero profits. Now assume that insurance companies just offer a single insurance policy to all customers. (We introduce the possibility of screening through a menu of insurance policies in the next section.) Given the premium $\pi$ the policy will be purchased by all the individuals whose expected value of damage is greater than or equal to this. That is, an individual will purchase the policy if $\pi(\theta) \geq \pi$ which using (1) is equivalent to $\theta \geq \frac{\pi}{1+\alpha}$. Thus to break even with zero profits, the premium must just equal the expected damage for those who choose to purchase the policy. Formally,

$$
\begin{aligned}
\pi & =E(\theta: \pi(\theta) \geq \pi) \\
& =E\left(\theta: \frac{\pi}{1+\alpha} \leq \theta \leq \Theta\right) \\
& =\frac{1}{1-F\left(\frac{\pi}{1+\alpha}\right)} \int_{\frac{\pi}{1+\alpha}}^{\Theta} \theta d F(\theta)
\end{aligned}
$$

Let $\pi^{*}$ be the corresponding equilibrium premium. This equilibrium is illustrated in Figure 1 for the uniform distribution of risks. ${ }^{6}$ It occurs where the curve $E(\theta: \pi(\theta) \geq \pi)$ crosses the $45^{\circ}$ line. The slope of the curve $E(\theta: \pi(\theta) \geq \pi)$ is inversely proportional to the degree of risk aversion and its intercept is the average risk equal to $\Theta / 2$. So increasing risk aversion reduces the equilibrium premium. It can be seen from the figure that insurance is only taken by those with high risks, namely $\theta \geq \frac{\Theta}{1+2 \alpha}$. This reflects the process of (partial) market unravelling through which only a fraction of the potential customers are actually served in equilibrium. The level of the premium is too high for the low risks (i.e., $\theta<\frac{\Theta}{1+2 \alpha}$ ) to find it worthwhile

\footnotetext{
${ }^{6}$ For a uniform distribution of risk, $E\left(\theta: \frac{\pi}{1+\alpha} \leq \theta \leq \Theta\right)=\frac{1}{2}\left[\frac{\pi}{1+\alpha}+\Theta\right]$ and the equilibrium premium then satisfies $\pi=\frac{1}{2}\left[\frac{\pi}{1+\alpha}+\Theta\right]$ which gives $\pi^{*}=\left(\frac{1+\alpha}{1+2 \alpha}\right) \Theta$.
} 
to take out the insurance. Since the equilibrium premium is decreasing with risk aversion, the number of those who opt out also decreases with risk aversion. On the other hand the market will completely unravel if individuals are risk neutral since the equilibrium premium is then equal to $\Theta$.

\section{[insert figure 1 about here]}

This market unravelling is a consequence of the fact that insurance companies cannot distinguish low-risk from high-risk consumers and when they offer the same premium to all consumers, the high-risk consumers force the premium up and this drives the low-risk out of the market. Of course, changing the distribution of risk will change the shape of $E(\theta: \pi(\theta) \geq \pi)$ and so the equilibrium premium. In fact, it is possible to conceive distribution functions $F(\theta)$ that lead to multiple equilibria in the sense that the curve $E(\theta: \pi(\theta) \geq \pi)$ crosses the $45^{\circ}$ line several times. At the low intersection point, the premium is low because a large fraction of the population is covered. At the high intersection point, the premium is high because only the small fraction of high-risk consumers take out the insurance. The important point is that whatever the equilibrium, the lowest risk individuals will always opt out (since $\pi(0)=0$ ) forcing the equilibrium premium above the average risk in the population $\left(\pi^{*}>\bar{\theta}\right)$. In this situation where adverse selection unravels the market, compulsory insurance based on average risk can make most consumers better off and thus be chosen by majority voting. The benefit of a compulsory insurance is that it can always force the lowest risk to participate lowering the equilibrium premium. In fact the equilibrium premium of a public (compulsory) insurance is $\pi^{\circ}=\bar{\theta}$. Because of the inevitable partial unravelling of a private (voluntary) insurance policy that offers the same premium to all consumers, we have that $\bar{\theta}<E\left(\theta: \frac{\pi}{1+\alpha} \leq \theta \leq \Theta\right)$ and 
thus for any equilibrium on the private market $\pi^{\circ}<\pi^{*}$.

We can now compare the payoff individuals get with either a full coverage private insurance or public insurance. At the risk of excessive simplification, we identify payoff as the difference between the premium a consumer is willing to pay given her risk (i.e., the value of insurance) less the premium effectively paid (i.e., the cost of insurance). Formally, the payoff of an individual with type $\theta$ and a private insurance policy with equilibrium premium $\pi^{*}$ is

$$
V\left(\pi^{*} ; \theta\right)=\operatorname{Max}\left\{0,(1+\alpha) \theta-\pi^{*}\right\}
$$

The payoff of an individual with type $\theta$ and a public insurance policy with equilibrium premium $\pi^{\circ}=\bar{\theta}$ is

$$
V\left(\pi^{\circ} ; \theta\right)=(1+\alpha) \theta-\pi^{\circ}
$$

Therefore since $\pi^{*}>\pi^{\circ}=\bar{\theta}$ we have

$$
\begin{array}{ll}
V\left(\pi^{*} ; \theta\right) \geq V\left(\pi^{\circ} ; \theta\right) & \text { for } \theta \leq \frac{\bar{\theta}}{1+\alpha} \\
V\left(\pi^{*} ; \theta\right)<V\left(\pi^{\circ} ; \theta\right) & \text { for } \theta>\frac{\bar{\theta}}{1+\alpha}
\end{array}
$$

Thus all the high-risk individuals with $\theta \geq \frac{\bar{\theta}}{1+\alpha}$ prefer public insurance while all the low-risk individuals with $\theta<\frac{\bar{\theta}}{1+\alpha}$ prefer private insurance. Hence we get the following result.

Proposition 1: (No screening) Suppose that (risk neutral and competitive) private companies offer the same insurance policy to all consumers, and that each individual degree of risk aversion is $\alpha>0$, then a majority

\footnotetext{
${ }^{7}$ In particular for the uniform distribution, we have $\pi^{\circ}=\frac{1}{2} \Theta<\frac{1+\alpha}{1+2 \alpha} \Theta=\pi^{*}$.
} 
of individuals prefer public (compulsory) to private (voluntary) insurance if and only if $\theta_{m} \geq \frac{\bar{\theta}}{1+\alpha}$.

Notice that this result holds true even for some positively skewed distributions of risk $\left(\theta_{m}<\bar{\theta}\right)$ leading the median to subsidise the high risk in the public insurance. Notice also that the political support for the public insurance increases with risk aversion. The reason is simple. All those participating in the private insurance market prefer a public insurance to benefit from a lower premium. Only those who opt out are worse off with a public insurance. More risk aversion induces more consumers to participate on the private insurance market and fewer to opt out, leading to a greater support for public insurance.

This finding is of course a consequence of the partial unravelling resulting from a voluntary participation in the private insurance market when insurance companies offer the same policy to all consumers. We know introduce the possibility for the insurance companies to offer different insurance policies to different consumers.

\section{Private insurance with screening}

We continue to assume a continuum of risk $\theta$ distributed on $[0, \Theta]$ according to the distribution function $F(\theta)$. The insurance companies offer a menu of insurance policies $\{\delta(\theta), \pi(\theta)\}_{\theta \in[0, \Theta]}$, where $\delta(\theta) \in[0,1]$ is the coverage rate and $\pi(\theta)$ is the premium intended for a consumer with risk $\theta$. In a fully separating equilibrium, each type $\theta$ selects the policy intended for her and the premium is actuarially fair $\pi(\theta)=\delta(\theta) \theta$. This requires to satisfy the following set of incentive compatibility constraints: For all $\theta \in[0, \Theta]$ and for all $\hat{\theta} \in[0, \Theta]$ with $\hat{\theta} \neq \theta$, 


$$
\begin{aligned}
& V(\delta(\theta), \pi(\theta) ; \theta)=(1+\alpha) \delta(\theta) \theta-\pi(\theta) \geq \\
& V(\delta(\widehat{\theta}), \pi(\widehat{\theta}) ; \theta)=(1+\alpha) \delta(\widehat{\theta}) \theta-\pi(\widehat{\theta})
\end{aligned}
$$

or given the actuarially fair premia,

$$
\begin{aligned}
& V(\delta(\theta), \pi(\theta) ; \theta)=(1+\alpha) \delta(\theta) \theta-\delta(\theta) \theta \geq \\
& V(\delta(\widehat{\theta}), \pi(\widehat{\theta}) ; \theta)=(1+\alpha) \delta(\widehat{\theta}) \theta-\delta(\widehat{\theta}) \widehat{\theta}
\end{aligned}
$$

To see whether there exists a separating equilibrium in the model, we follow the incentive compatibility approach of Mailath (1987). A necessary condition for a separating equilibrium is that the following local incentive compatibility conditions hold: for all $\theta \in[0, \Theta]$

$$
\begin{aligned}
{\left[\frac{\partial V(\delta(\hat{\theta}), \pi(\hat{\theta}) ; \theta)}{\partial \hat{\theta}}\right]_{\hat{\theta} \rightarrow \theta} } & =(1+\alpha) \delta^{\prime}(\theta) \theta-\delta^{\prime}(\theta) \theta-\delta(\theta)=0 \\
& =\alpha \theta \delta^{\prime}(\theta)-\delta(\theta)=0
\end{aligned}
$$

In other words, any separating equilibrium requires that no type be able to obtain a strictly higher payoff by announcing another arbitrary close type. From Mailath (1987), we also see that the payoff functions are such that these local necessay conditions are also sufficient. Hence the equilibrium coverage rate function $\delta(\theta)$ is the solution to the following homogeneous first-order differential equation,

$$
\delta^{\prime}(\theta)=\frac{\delta(\theta)}{\alpha \theta}
$$


Solving this equation we get,

$$
\begin{aligned}
\delta(\theta) & =C e^{\int \frac{1}{\alpha \theta} d \theta} \\
& =C e^{\frac{\log \theta}{\alpha}} \\
& =C \theta^{1 / \alpha}
\end{aligned}
$$

where $C$ is a constant of integration. Using the terminal condition $\delta(\Theta)=1$ we get $C=\Theta^{-1 / \alpha}$ and the equilibrium coverage function is,

$$
\delta^{*}(\theta)=\left(\frac{\theta}{\Theta}\right)^{1 / \alpha}
$$

with the corresponding equilibrium premium function,

$$
\pi^{*}(\theta)=\delta^{*}(\theta) \theta=\theta\left(\frac{\theta}{\Theta}\right)^{1 / \alpha}
$$

Hence the policy menu $\left\{\delta^{*}(\theta), \pi^{*}(\theta)\right\}_{\theta \in[0, \Theta]}$ characterizes the unique separating equilibrium in this continuous-type insurance model. Substituting the equilibrium insurance policies into the payoff functions we get

$$
\begin{aligned}
V\left(\delta^{*}(\theta), \pi^{*}(\theta) ; \theta\right) & =(1+\alpha) \delta^{*}(\theta) \theta-\pi^{*}(\theta) \\
& =\alpha \theta\left(\frac{\theta}{\Theta}\right)^{1 / \alpha}
\end{aligned}
$$

We assume that the public insurance policy takes the following form,

$$
\left\{\delta^{\circ}=1, \pi^{\circ}=\bar{\theta}\right\}
$$

The public insurance policy charges a uniform premium based on average risk and imposes full coverage. So the payoff functions for the public insurance are exactly the same as in the previous section. We can now derive the marginal type $\theta=\theta^{\circ}$ who is indifferent between the public and the private insurance 
policies. Obviously all low-risk consumers with $\theta<\theta^{\circ}$ will prefer the private separating insurance policy and all high-risk consumers with $\theta \geq \theta^{\circ}$ will prefer the full coverage public insurance policy. Given $(3), \theta=\theta^{\circ}$ solves

$$
\alpha \theta\left(1-\left(\frac{\theta}{\Theta}\right)^{1 / \alpha}\right)=\bar{\theta}-\theta
$$

Since the LHS is always positive, the marginal type $\theta^{\circ}$ must be less than the average $\bar{\theta}$. Unfortunately it is not possible to obtain an explicit solution for $\theta^{\circ}$. Numerical solutions for $\theta^{\circ}$ are given in Table 1 for various levels of risk aversion and average risk. Table 1 reveals that for any given the average risk, the marginal type is decreasing with risk aversion and thus that the political support for public insurance over private separating insurance increases with risk aversion (as in the previous section). Moreover Table 1 shows that the marginal type is significantly below the average type so that even for some positively skewed distributions of risk, a majority will support full public insurance over private separating insurance.

[insert Table 1 about here]

The following proposition summarizes our result with private separating insurance policies.

Proposition 2: (Screening) Suppose that (risk neutral and competitive) private companies offer fully separating insurance policies in equilibrium to all consumers, and that the public insurance contract is the same for all consumers, then there exists a critical risk level $0<\theta^{\circ}<\bar{\theta}$ such that a majority of consumers prefer the public insurance if and only if $\theta_{m} \geq \theta^{\circ}$. Moreover $\theta^{\circ}$ is decreasing with the degree of risk aversion, $\alpha$.

We emphasize that this Proposition is general and does not depend on the 
distribution of risk in the population. More risk aversion increases the political support for public insurance because the adverse selection problem induces the private insurance companies to provide only partial coverage to low risk individuals. So a greater risk aversion reduces the attractiveness of private insurance and leads more individuals to favour public insurance with full coverage (although at a higher price for the low risk).

\section{Top-up insurance}

In this section we introduce the possibility of mixing public and private insurance. A basic result in the expected utility framework is that a mixed insurance can make both the low risk and the high risk individuals better off (e.g. Eckstein et al, 1985). The reason is that partial public insurance operates a cross-subsidy from the low-risk to the high risk thereby relaxing the incentive constraint on the private insurance market. Figure 2a illustrates this result for two risk types (assuming low risk individuals are in a majority). It shows that even if the low risk individuals do not favour full public insurance, they may prefer a mixed insurance to the pure private system. We show there cannot be a majority for such a mixture of private and public insurance under the dual theory of risk aversion. Our analysis thus suggests that the political support for a mixed system of insurance rests heavily on the modelling of choice under uncertainty. At one extreme the expected utility theory is the most favorable to mixing public and private insurance while at the other extreme the dual theory tends to favour separation between private and public insurance.

Let $\delta \in[0,1]$ and $\pi=\delta \bar{\theta}$ be the public insurance policy. Individuals can freely complement this public insurance with a private insurance leading to a total coverage for type $\theta$ equal to $\delta(\theta)$ where the extra private coverage 
$\delta(\theta)-\delta$ is purchased at a fair price $(\delta(\theta)-\delta) \theta$ and satisfies the incentive constraints. The corresponding payoff functions are,

$$
\begin{aligned}
V(\delta, \delta(\theta), \pi(\theta) ; \theta) & =(1+\alpha) \delta \theta-\delta \bar{\theta}+(1+\alpha)(\delta(\theta)-\delta) \theta-(\delta(\theta)-\delta) \theta \\
& =\delta(\theta-\bar{\theta})+\alpha \delta(\theta) \theta
\end{aligned}
$$

In any separating equilibrium, the following necessary local incentive compatibility condition must hold: for all $\theta \in[0, \Theta]$

$$
\begin{aligned}
{\left[\frac{\partial V(\delta, \delta(\hat{\theta}), \pi(\hat{\theta}) ; \theta)}{\partial \hat{\theta}}\right]_{\hat{\theta} \rightarrow \theta} } & =(1+\alpha) \delta^{\prime}(\theta) \theta-\delta^{\prime}(\theta) \theta-(\delta(\theta)-\delta)=0 \\
& =\alpha \theta \delta^{\prime}(\theta)-(\delta(\theta)-\delta)=0
\end{aligned}
$$

From Mailath(1987), it is easily seen that the payoff functions are such that the local incentive compatibility condition is also sufficient, implying that the solution to the equation above charaterizes the separating equilibrium for the continuum-type case. Hence the separating equilibrium coverage rate function $\delta(\theta)$ solves the following non-homogeneous linear first-order differential equation,

$$
\delta^{\prime}(\theta)=\frac{\delta(\theta)}{\alpha \theta}-\frac{\delta}{\alpha \theta}
$$

The general solution is,

$$
\begin{aligned}
\delta(\theta) & =e^{\int \frac{1}{\alpha \theta} d \theta}\left(C(\delta)-\int \frac{\delta e^{-\int \frac{1}{\alpha \theta} d \theta}}{\alpha \theta} d \theta\right) \\
& =C(\delta) \theta^{1 / \alpha}-\theta^{1 / \alpha} \int \frac{\delta \theta^{-1 / \alpha}}{\alpha \theta} d \theta \\
& =C(\delta) \theta^{1 / \alpha}-\frac{\delta \theta^{1 / \alpha}}{\alpha} \int \theta^{-(1+\alpha) / \alpha} d \theta \\
& =C(\delta) \theta^{1 / \alpha}+\delta
\end{aligned}
$$


where $C(\delta)$ is the constant of integration given the public coverage rate. Setting $\delta(\Theta)=1$ for a Pareto optimal equilibrium, we get $C(\delta)=(1-\delta) \Theta^{-1 / \alpha}$. Therefore, the unique separating equilibrium is,

$$
\delta^{*}(\theta)=(1-\delta)\left(\frac{\theta}{\Theta}\right)^{1 / \alpha}+\delta
$$

where $\left(\frac{\theta}{\Theta}\right)^{1 / \alpha}<1$ represents the inherent partial coverage resulting from the adverse selection problem on the private insurance market. In particular, setting $\delta=0$ we get the same expression for $\delta^{*}(\theta)$ as in the previous section. Notice that in equilibrium, for all types total coverage is increasing with the public coverage rate $\delta$ since $\left(\frac{\theta}{\Theta}\right)^{1 / \alpha}<1$.

Plugging this equilibrium policy into the payoff function we get

$$
\begin{aligned}
V\left(\delta, \delta^{*}(\theta), \pi^{*}(\theta) ; \theta\right) & =\alpha \delta^{*}(\theta) \theta+\delta(\theta-\bar{\theta}) \\
& =\alpha\left[(1-\delta)\left(\frac{\theta}{\Theta}\right)^{1 / \alpha}+\delta\right] \theta+\delta(\theta-\bar{\theta})
\end{aligned}
$$

We are now in a position to determine the equilibrium level of public coverage $\delta \in[0,1]$. Differentiating the above payoff functions with respect to $\delta$ we get,

$$
\frac{\partial V(.)}{\partial \delta}=(\theta-\bar{\theta})+\alpha \theta\left[1-\left(\frac{\theta}{\Theta}\right)^{1 / \alpha}\right]
$$

Interestingly, this expression is independent of $\delta$. Moreover it is easily seen that there exists a marginal type $\theta^{\circ}<\bar{\theta}$ who is indifferent to a change in the public coverage rate $\delta$. Notice that this marginal type is the same as in the previous section where public coverage was set equal to one. ${ }^{8}$ This

\footnotetext{
${ }^{8}$ Therefore, the value of the marginal type as a function of $\alpha$ and $\bar{\theta}$ is the same as indicated in Table 1.
} 
is because the preference for public coverage is independent of the level of public coverage. Finally, notice that all those with risk $\theta<\theta^{\circ}$ prefer no public coverage and all those with risk $\theta \geq \theta^{\circ}$ prefer full public coverage. Therefore either the median risk is below $\theta^{\circ}$ and the political equilibrium involves no public insurance at all, or alternatively the median risk is above $\theta^{\circ}$ and the political equilibrium involves full public insurance. It follows that a mixed system is not politically sustainable. We summarize our results in the following proposition.

Proposition 3: (Policy mix) Suppose that (risk neutral and competitive) private companies offer fully separating insurance policies in equilibrium to all consumers to complement a public insurance contract which is the same for all consumers, then there exists a critical risk level $0<\theta^{\circ}<\bar{\theta}$ such that a majority of consumers prefer a full public insurance if and only if $\theta_{m} \geq \theta^{\circ}$ and no public insurance at all otherwise. A mixture of public and private insurance is not politically sustainable. Moreover since $\theta^{\circ}$ is decreasing with the degree of risk aversion ( $\alpha$ ), the political support for a full public insurance increases with risk aversion.

The dual theory, because of its linearity property, tends to produce corner solutions in optimization problems. This is why we get either full public insurance or pure private insurance. Figure 2 illustrates why a mixed system is more likely under expected utility than within the dual theory. ${ }^{9}$

\section{[Insert figure 2 about here]}

Although such corner solutions may look awkward, their comparative statics properties are intuitive. Recall that under dual theory,a majority

\footnotetext{
${ }^{9} \mathrm{I}$ am indebted to one anonymous referee for suggesting me this graphical comparison.
} 
prefer public insurance if $\theta_{m} \geq \theta^{\circ}$ and private insurance if $\theta_{m}<\theta^{\circ}$. The parameter $\theta^{\circ}$ therefore acts as a measure of the collective preference for public insurance: with a decrease in $\theta^{\circ}$ tending to encourage public insurance and an increase in $\theta^{\circ}$ tending to inhibit public insurance. Thus it is of interest to see how $\theta^{\circ}$ is affected by the underlying parameters of the model. From (4) we note that $\theta^{\circ}$ depends on one hand on risk aversion $\alpha$ and on the other hand on the average risk $\bar{\theta}$. Increasing risk aversion, as we might expect, makes the better coverage of public insurance more appealing and thus raises the political support for private insurance. Increasing the average risk raises the price of public insurance making public insurance relatively less attractive.

The result that there cannot be a majority support for a mixed system is not to be taken as a general feature of the dual theory. It is easy to think of a more complex model in which corner solutions no longer prevail. This is for instance the case in the multivariate version of the theory (see Yaari, 1986). As we show in Appendix B a mixed system can also prevail if we introduce a slight distortion in public insurance. ${ }^{10}$ Moreover it is shown that the equilibrium degree of public insurance is increasing with risk aversion and the median risk but decreasing with average risk and the intensity of the distortion.

\section{$5 \quad$ Testing for risk and the demand for public insurance}

In this section we show how the recent progress in the information technology that improves the capacity of private insurance companies to infer individual risks will affect the demand for public insurance (for a survey on the effects of

\footnotetext{
${ }^{10}$ Indeed, one might expect that compulsory public insurance is more amenable to distortion than voluntary private insurance.
} 
genetic screening, see Barr, forthcoming ). Suppose that private companies have access to some genetic tests that reveal the true risk of any individual with probability $s \in[0,1]$ and reveal nothing at all with probability $1-s$. So $s$ is a measure of the quality of the information available to private companies in using this technology. We assume that if the test is successfull private companies offer a contract with actuarially fair premium and full coverage giving a payoff $(1+\alpha)-\theta$ to an individual with risk $\theta$, otherwise they offer an incentive compatible contract such as described in Section 3 giving a payoff as in (3). So the expected payoff for an individual with risk $\theta$ resulting from this private insurance policy contingent on a test of quality $s$ is ${ }^{11}$

$$
E V\left(\delta^{*}(\theta), \pi^{*}(\theta) ; \theta, s\right)=s \alpha \theta+(1-s) \alpha \theta\left(\frac{\theta}{\Theta}\right)^{1 / \alpha}
$$

The payoff resulting from the public insurance policy is unchanged by the introduction of this test. Comparing both payoff we can derive the individual with risk $\theta=\theta_{s}^{\circ}$ who is indifferent between both systems. This is given by the solution to

$$
\alpha \theta\left[1-\left(s+(1-s)\left(\frac{\theta}{\Theta}\right)^{1 / \alpha}\right)\right]=\bar{\theta}-\theta
$$

It is easily seen that the solution of this equation, $\theta_{s}^{\circ}$, is increasing in the quality of the test $s$ and tends to $\bar{\theta}$ when $s$ approaches $s=1$. Since all those with risk $\theta \geq \theta_{s}^{\circ}$ prefer public insurance, it turns out that the demand for public insurance decreases as the quality of the test improves. We state this result in the next proposition.

\footnotetext{
${ }^{11}$ To be more precise this expected payoff should be deflated by a risk premium to reflect the risk associated to the imperfection of the test. However this will complicate the analysis without bringing more insights and we prefer to ignore it
} 
Proposition 4: (testing) Suppose that (risk neutral and competitive) private companies offer fully separating insurance policies in equilibrium to every consumer whose risk has not been revealed by the test (with probability $(1-s)$ ), and a full coverage at actuarially fair price to every consumer whose risk has been revealed by the test (with probability s). If the public insurance contract is the same for all consumers, then there exists a critical risk level $0<\theta_{s}^{\circ} \leq \bar{\theta}$ such that a majority of consumers prefer the public insurance if and only if $\theta_{m} \geq \theta_{s}^{\circ}$. Moreover $\theta_{s}^{\circ}$ is decreasing with the degree of risk aversion, $\alpha$ and increasing with the quality of the test $s$.

The main implication of Proposition 4 is that the demand for public insurance decreases with the increasing capacity of private companies in assessing individual risks up to a point where public insurance may no longer be politically sustainable.

\section{Conclusion}

In this paper we have derived the circumstances under which a majority prefers public insurance over private insurance in the presence of adverse selection problem. The main results are that there is in general a majority support for public insurance and that our non-expected utility framework (risk aversion without diminishing marginal utility) is less favourable to mixing private and public insurance than the expected utility setting. This seems to be consistent with aspects observed in the real world. For example health care is mostly provided by government in many countries. We have also shown that the demand for public insurance increases with risk aversion and decreases with the quality of the tests used by private companies to assess individual risks. 
The results are of course illustrative and not conclusive, but still they are instructive. The limitations of our analysis need to be emphasized. First we have not considered the moral hazard problem. Second we have ignored the important agency problem. This agency problem is specially acute in health care where the insured and uninformed patient cannot monitor the prescribing agent who does not bear the cost of prescribtion but on the contrary may benefit from over-prescibtion. The nature of the moral hazard and agency problem is the same for a private insurer as for a public insurer. To do justice to these crucial problems would deserve a separate paper, but would probably not affect our results about the relative preference for public and private insurance. The third and perhaps most important limitation of our analysis is the absence of distributive argument for public insurance. The reason was to isolate the insurance motive for public insurance and show that public insurance can be supported by a majority without resorting to distributive motive. However we agree that most systems of health care and state pensions in the European Union currently include a potential amount of income redistribution by the mere fact that contributions are income-related. We to take up this issue in a separate paper in which individuals differ not only in their risk but also in their income (De donder and Hindriks, 2000). The model is similar to Rochet (1991) or Cremer and Pestieau (1996) with the difference that we use the non-expected utility framework and majority voting. The key question is of course how the the degree of redistribution of public insurance and the correlation between income and risk affect the political equilibrium outcome.

Acknowledgements. This paper has been prepared during my visit at CORE. I thank Jacques Dreze for perceptive discussions and Michel Lebreton and Pierre Pestieau for useful comments. I am also very grateful to seminar participants at IFS (UCL, London), Leuven and Marseilles. Special thanks 
go to one Co-Editor and two anonymous referees for their valuable help. Financial support from CORE is gratefully acknowledged.

\section{Appendix}

\section{Appendix A: Reservation premium in the dual theory}

Let $X$ be a random variable with the cumulative distribution function $F(x)$ on $[\underline{x}, \bar{x}]$ and let $U(X)$ be the utility assigned to $X$ under the dual theory. Following Yaari (1987) we have

$$
U(X)=\int x \phi^{\prime}(G(x)) d F(x)
$$

where $G(x)=1-F(x)$ is the decumulative distribution function (with $G(\underline{x})=1$ and $G(\bar{x})=0$ ) and $\phi^{\prime}($.$) are non-negative weigths adding up$ to 1.Risk aversion is characterized by the convexity of $\phi$. If $\phi$ is convex then bad outcomes (with high $G(x)$ ) receives relatively high weights whereas good outcomes (with low $G(x)$ ) receive relatively low weights. So, $U(X)$ is the certainty equivalent of $X$ defined as the mean in which probabilities of bad outcomes are increased and probabilities of good outcomes are decreased. Since $U$ is linear in $x$, attitude towards risk is separated from attitude towards wealth. We can now apply this dual theory in our simple two-state of nature insurance framework. For an individual with income $w>1$ facing a damage $d=1$ with probability $\theta \in[0, \Theta]$ (with $\Theta<1$ ), insurance contract with premium $\pi \geq 0$ and coverage rate $\delta \in[0,1]$ yields the random variable $X=(w-\pi-(1-\delta), \theta ; w-\pi ; 1-\theta)$ whereas no insurance yields the random variable $X_{0}=(w-1, \theta ; w ; 1-\theta)$. Under Yaari's Dual theory, the reservation premium $\pi=\pi(\theta)$ is defined by

$$
U(X)=\phi(\theta)[w-\pi-(1-\delta)]+(1-\phi(\theta))[w-\pi]=
$$




$$
U\left(X_{0}\right)=\phi(\theta)[w-1]+(1-\phi(\theta))[w]
$$

where risk aversion is represented by $\phi(\theta)>\theta$ (and $1-\phi(\theta)<1-\theta)$. Letting $\phi(\theta)=(1+\alpha) \theta \quad$ (where $\alpha \geq 0$ denotes the degree of risk aversion) and rearranging the above expression we get the following reservation premium.

$$
\pi(\theta)=(1+\alpha) \theta \delta
$$

where $0 \leq \alpha \leq \frac{1-\Theta}{\Theta} \quad$ (the upper bound guarantees that $\phi(\theta) \leq 1 \forall$ $\theta \in[0, \Theta])$.

\section{Appendix B: Policy mix with distortionary public insurance}

Let us consider, for example, that the public insurance premium for any coverage rate $\delta \in[0,1]$ is

$$
\pi_{\mu}^{\circ}=(1+\mu \delta) \bar{\theta} \delta
$$

where the deadweight loss is proportional to $\mu \delta^{2} \geq 0$. (Recall that the deadweight loss from taxation is approximately proportional to the square of the tax rate.) The parameter $\mu \geq 0$ measures the intensity of the deadweight loss, with non-distortionary public insurance if $\mu=0$. It is easily seen that the equilibrium on the private market is unaffected by the deadweight loss of public insurance (i.e., $\left.\delta^{*}(\theta)=(1-\delta)\left(\frac{\theta_{m}}{\Theta}\right)^{1 / \alpha}+\delta\right)$. It follows that the payoff of public coverage $\delta$ for an individual with risk $\theta$ is

$$
\begin{aligned}
V_{\mu}\left(\delta, \delta^{*}(\theta), \pi^{*}(\theta) ; \theta\right) & =\alpha \delta^{*}(\theta) \theta+\delta\left(\theta-\pi_{\mu}^{\circ}\right) \\
& =\alpha \theta\left[(1-\delta)\left(\frac{\theta_{m}}{\Theta}\right)^{1 / \alpha}+\delta\right]+\delta(\theta-\bar{\theta})-\mu \delta^{2} \bar{\theta}
\end{aligned}
$$

which is strictly concave in $\delta$ for any $\mu>0$. Therefore the deadweight loss from public insurance tends to produce interior solutions for the individually 
optimal degree of public insurance. Moreover all individual have singlepeaked preferences over public coverage $\delta$ and the high risk individuals prefers more public insurance than the low risk individuals. By the median voter theorem, the majority voting outcome is characterized by the degree of public insurance that is optimal for the median risk individual $\theta=\theta_{m}$. From the first-order condition for the median voter

$$
\frac{\partial V_{\mu}\left(. ; \theta_{m}\right)}{\partial \delta}=\alpha \theta_{m}\left(1-\left(\frac{\theta_{m}}{\Theta}\right)^{1 / \alpha}\right)+\left(\theta_{m}-\bar{\theta}\right)-2 \mu \delta \bar{\theta} \leq 0
$$

with strict equality for $\delta>0$. Solving we get

$$
\begin{aligned}
\delta_{m}=0 & \text { if } \theta_{m} \leq \theta^{\circ} \\
\delta_{m}=\frac{\alpha \theta_{m}\left(1-\left(\frac{\theta_{m}}{\Theta}\right)^{1 / \alpha}\right)+\left(\theta_{m}-\bar{\theta}\right)}{2 \mu \bar{\theta}} & \text { if } \theta_{m}>\theta^{\circ} \text { and } \mu>\mu^{\circ} \\
\delta_{m}=1 & \text { if } \theta_{m}>\theta^{\circ} \text { and } \mu \leq \mu^{\circ}
\end{aligned}
$$

where $\theta^{\circ}$ is defined implicitely by (4) and $\mu^{\circ}=\frac{\alpha \theta_{m}\left(1-\left(\frac{\theta_{m}}{\Theta}\right)^{1 / \alpha}\right)+\left(\theta_{m}-\bar{\theta}\right)}{2 \bar{\theta}}$ (with $\mu^{\circ} \geq 0$ for all $\left.\theta_{m} \geq \theta^{\circ}\right)$. Therefore the majority voting outcome involves a mixed system $\left(0<\delta_{m}<1\right)$ if (i) the median risk is high enough (making some public insurance desirable to all those above the median risk) and (ii) the deadweight loss increases at a sufficiently high rate with public coverage (making full public insurance non-desirable to all those below the median risk). The comparative statics properties of the interior solution are the following. Increased risk aversion increases the degree of public coverage $\left(\partial \delta_{m} / \partial \alpha>0\right)$. The reason is that a more risk averse median voter is more sensitive to the fact that public insurance can offer a better coverage than private insurance. Increasing the median risk leads to more public insurance $\left(\partial \delta_{m} / \partial \theta_{m}>0\right)$ while increasing the average risk increases the price of public insurance and leads to less public insurance $\left(\partial \delta_{m} / \partial \bar{\theta}<0\right)$. Lastly the higher the deadweight loss, the lower the demand for public insurance $\left(\partial \delta_{m} / \partial \mu<0\right)$. To sum up 
Proposition 3*: (Policy mix with distortion) When public insurance is distortionary and the deadweight loss increases sufficiently rapidly with public coverage, then the majority voting outcome involves partial public insurance if $\theta_{m} \geq \theta^{\circ}$ and no public insurance at all otherwise. In the mixed system, the equilibrium degree of public insurance is an increasing function of risk aversion and median risk, and a decreasing function of deadweight loss and average risk.

\section{References}

[1] Barr, N., The Welfare State as Piggy Bank, unpublished manuscript.

[2] Besley, T. and M. Gouveia, 1994, Alternative systems of health care provision, Economic Policy, 19, 199-258.

[3] Cremer, H. and P. Pestieau, 1996, Redistributive taxation and social insurance, International Tax and Public Finance, 3, 281-95.

[4] De Donder, P. and J. Hindriks, 2000, The politics of redistributive social insurance, mimeo CORE, U. catholique de Louvain.

[5] Doherty N. and L. Eeckhoudt, 1995, Optimal insurance without expected utility: the dual theory and the linearity of insurance contracts, Journal of Risk and Uncertainty, 10, 157-79.

[6] Eckstein, Z., M. Eichenbaum and D. Peled, 1985, Uncertain lifetimes and the welfare enhancing properties of annuity market and social security, Journal of Public Economics, 26, 303-26.

[7] Epple, D. and R. Romano, 1996, Public provision of private goods. Journal of Political Economy, 104, 57-84. 
[8] Gouveia, M., 1997, Majority rule and the public provision of a private good, Public Choice, 93, 221-44.

[9] Hindriks, J. and G.D. Myles, Intermediate Public Economics. Cambridge University Press. Forthcoming.

[10] Karni,E. and I. Zilcha, 1986, Welfare and comparative statics implications of fair social security, Journal of Public Economics, 30, 341-57.

[11] Lynn Coronado, J., D. Fullerton and T. Glass, 2000, The progressivity of social security, NBER Working Paper no 7520.

[12] Machina, M., 1995, Non-expected utility and the robustness of the classical insurance paradigm, Geneva Papers on Risk and Insurance Theory, 20, 9-50.

[13] Mailath, G., 1987, Incentive compatibility in signaling games with a continuum of types, Econometrica, 55, 1349-65.

[14] Mas-colell, A., M.D. Whinston and J. Green, 1995, Microeconomic Theory, Oxford University Press: New York.

[15] Miles, D. and A. Timmermann, 1999, Risk sharing and transition costs in the reform of pension systems in Europe, Economic Policy, 29, 253-86.

[16] OECD, 1994, The reform of health care systems- a review of seventeen OECD countries, Health Policy Studies no 5, Paris: OECD.

[17] Rochet, J.-C., 1991, Incentives, redistribution and social insurance, The Geneva Papers on Risk and Insurance Theory, 16, 143-65.

[18] Rothschild, M. and J.E. Stiglitz, 1976, Equilibrium in competitive insurance markets: an essay in the economics of incomplete information, Quarterly Journal of Economics, 90, 624-49. 
[19] Yaari, M., 1986, Univariate and multivariate comparisons of risk aversion: a new approach, in Essays in Honor of Kenneth Arrow, ed. by W.P. Heller, R. Starr, and D. Starrett. Cambridge: Cambridge University Press.

[20] Yaari, M., 1987, The dual theory of choice under risk, Econometrica, 55, 95-115. 
Figure 1 Partial market unravelling with uniform distribution of risk

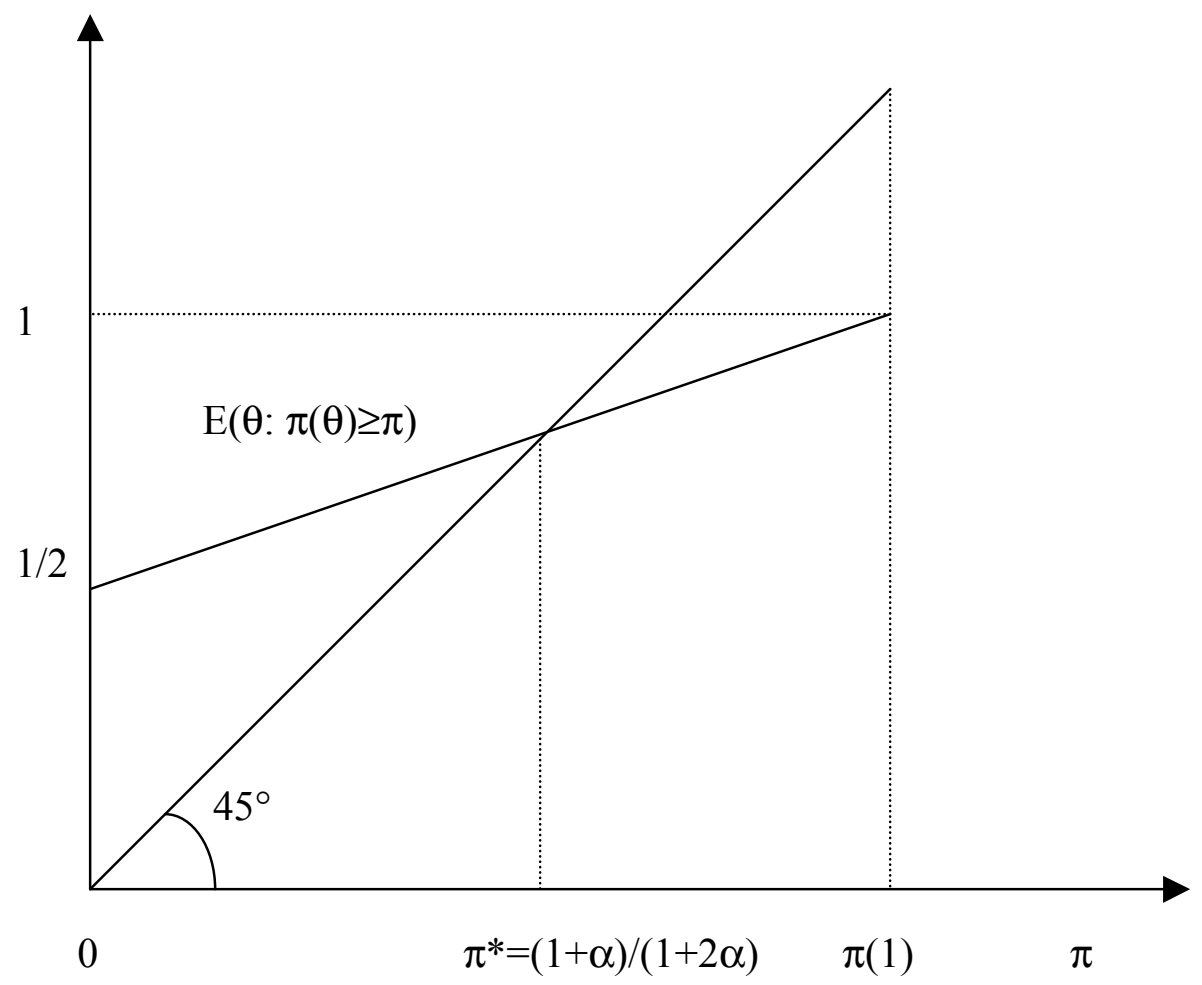


Table 1. Critical risk level $\theta^{\circ}$ as a function of risk aversion $\alpha$ and average risk.

\begin{tabular}{|c|c|c|c|c|c|}
\hline \multirow[t]{2}{*}{$\alpha$} & \multicolumn{4}{|c|}{ Average risk } & \\
\hline & 0.25 & 0.33 & 0.5 & 0.66 & 0.75 \\
\hline 0.25 & 0.2 & 0.27 & 0.4 & 0.54 & 0.62 \\
\hline 0.33 & 0.19 & 0.25 & 0.38 & 0.52 & 0.6 \\
\hline 0.5 & 0.17 & 0.23 & 0.35 & 0.48 & 0.56 \\
\hline 0.66 & 0.15 & 0.21 & 0.32 & 0.46 & 0.53 \\
\hline 0.75 & 0.14 & 0.2 & 0.31 & 0.45 & 0.52 \\
\hline 1 & 0.13 & 0.18 & 0.29 & 0.42 & 0.5 \\
\hline 1.33 & 0.12 & 0.17 & 0.27 & 0.4 & 0.48 \\
\hline 1.5 & 0.11 & 0.16 & 0.27 & 0.39 & 0.47 \\
\hline 1.66 & 0.11 & 0.16 & 0.26 & 0.39 & 0.46 \\
\hline 1.75 & 0.11 & 0.15 & 0.26 & 0.38 & 0.46 \\
\hline 2 & 0.1 & 0.15 & 0.25 & 0.37 & 0.45 \\
\hline 3 & 0.09 & 0.14 & 0.23 & 0.36 & 0.43 \\
\hline 4 & 0.09 & 0.13 & 0.22 & 0.34 & 0.42 \\
\hline 5 & 0.08 & 0.12 & 0.21 & 0.33 & 0.41 \\
\hline
\end{tabular}




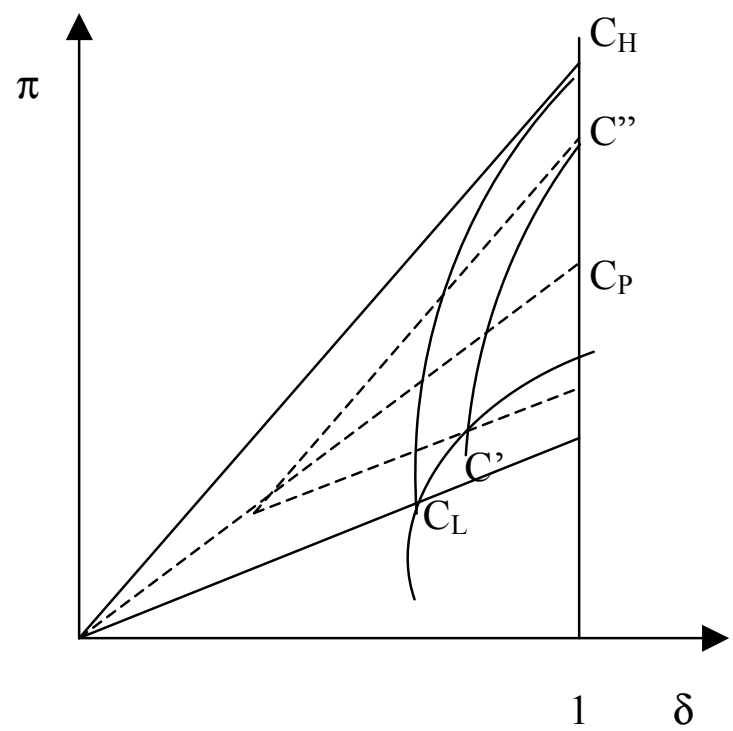

a. expected utility theory

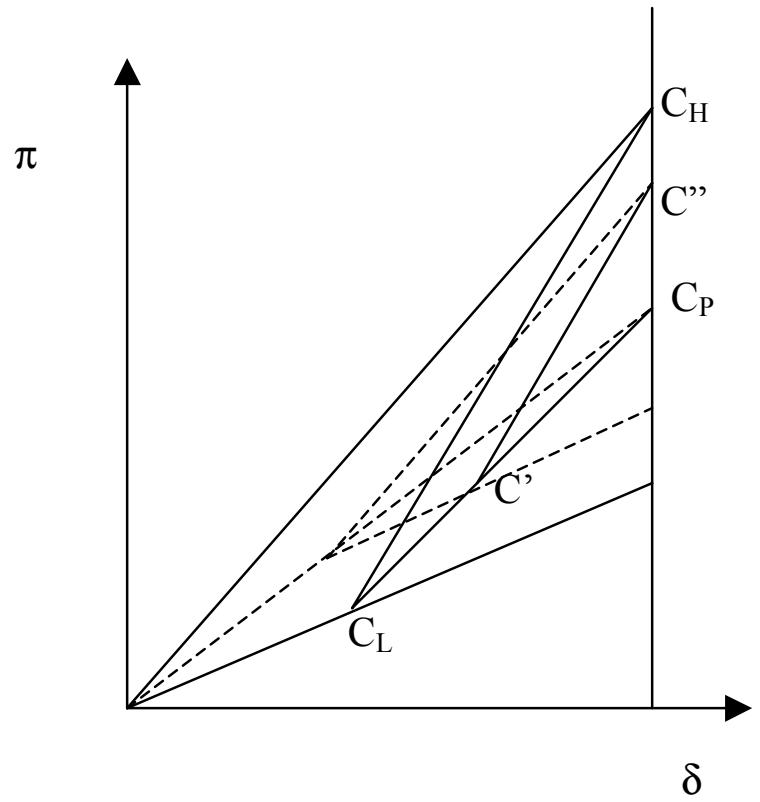

b. dual theory

Figure 2. Mixed insurance in two-type model. (a) expected utility theory: the separating equilibrium without public insurance is given by contract $C_{L}$ for the low risk type and $C_{H}$ for the high risk type (both contracts break even and are incentive compatible). A pure public insurance system is given by a full coverage contract $C_{P}$ with average risk premium. With a majority of low risk types full public insurance cannot be a majority voting outcome. However there is a unanimous support for a mixture of public and private insurance leading to either $\mathrm{C}^{\prime}$ for the low risks (who prefer $\mathrm{C}^{\prime}$ to $\mathrm{C}_{\mathrm{L}}$ ) or $\mathrm{C}$ " for the high risks (who prefer $\mathrm{C}$ " to $\mathrm{C}_{\mathrm{H}}$ ). (b) dual theory: the indifference curves are linear which implies that if low risks are indifferent between pure private insurance $C_{L}$ and full public insurance $C_{P}$, then they are also indifferent between any mixture of public and private insurance. 


\section{Q Queen Mary \\ University of London}

This working paper has been produced by the Department of Economics at Queen Mary, University of London

Copyright ( 2001 Jean Hindriks All rights reserved.

\section{Department of Economics}

Queen Mary, University of London

Mile End Road

London E1 4NS

Tel: +44 (0)20 78825096 or Fax: +44 (0)20 89833580

Email: j.conner@qmw.ac.uk

Website: www.econ.qmw.ac.uk/papers/wp.htm 